\title{
TRIANGULAR PERMUTATION NUMBERS
}

\section{JOHN RIORDAN}

1. Introduction. The numbers of the title are those treated in [4], ${ }^{1}$ that is, the numbers of permutations of $n$ elements with $x$ elements in forbidden positions specified by a triangular array which is isosceles and of side $n$ (for example, element $i$ forbidden positions $i$ to $n$ ). They are also, as noted in [4], the numbers of permutations with $x$ ascending runs, or what is the same thing, the numbers with $x-1$ falls, a fall being a succession $i j$ with $i>j$. Also they have occurred in other apparently unrelated contexts, in various expansions in the older literature noted by von Schrutka [8], in statistics (Dwyer [1] and [2]), and in operational calculus (Gardner and Barnes [3, p. 295]).

A recent classification of permutations used by Sprague [7] according to number of readings left to right necessary to put all elements in standard order is another instance where the numbers appear. The quickest way to see this is to notice (cf. Sade [5]) that if a permutation takes $x$ readings, then its conjugate, which has elements and positions interchanged, has $x$ rising sequences.

Nevertheless a direct attack on the enumeration by readings has an independent interest because of including in a combinatorial setting some of the apparently unrelated results.

2. The number of permutations with $x$ readings. Write $r(x, n)$ for the number of permutations of $n$ elements taking $x$ readings and

$$
R_{n}(t)=\sum r(x, n) t^{x}
$$

for its generating function. Summation is over all possible values which are clearly 1 to $n$, and can be left indefinite because $r(x, n)$ is zero elsewhere.

It is clear that $r(1, n)=r(n, n)=1$, the corresponding single permutations being $1,2, \cdots, n$ and $n, n-1, \cdots, 1$. These are complements of each other (sum of elements in any position being $n+1)$ and more generally, by a simple argument, $r(x, n)=r(n+1$ $-x, n)$; the numbers for fixed $n$ are symmetrical. Hence $R_{1}(t)=t$, $R_{2}(t)=t+t^{2} ; R_{0}(t)$ has of course no sense combinatorially, but may be fixed by a usual convention as unity.

The recurrence for falls (cf. [8]) is by position of the added ele-

Received by the editors May 26, 1950 .

${ }^{1}$ Numbers in brackets refer to the bibliography at the end of the paper. 
ment $n$ and works easily; the same thing for readings is awkward (but cf. Sade [6]) and following the hint given by conjugacy we consider instead recurrence by elements in the added position $n$.

Let $R_{n}^{(k)}$ be the generating function for readings with element $k$ in position $n$, so that

$$
R_{n}=R_{n}^{(1)}+R_{n}^{(2)}+\cdots+R_{n}^{(n)} .
$$

Then $R_{n}^{(1)}=t R_{n-1}$, since element 1 takes one reading independently of all other elements. Also $R_{n}^{(n)}=R_{n-1}$ since element $n$ in position $n$ must be included in a reading with element $n-1$. In general

$$
R_{n}^{(k)}=C_{n-1, k-1} R_{k-1} R_{n-k}, \quad k>1,
$$

since for $k$ in position $n$, the elements are divided into two sets containing, respectively, elements 1 to $k-1$ and $k+1$ to $n$, and these are independent with respect to readings; the binomial coefficient enumerates the number of ways positions may be assigned to the elements of one set (and hence to the other).

Hence finally

$$
\begin{aligned}
R_{n}= & (t-1) R_{n-1}+R_{n-1}+(n-1) R_{1} R_{n-2}+\cdots \\
& +C_{n-1, k} R_{k} R_{n-1-k}+\cdots+R_{n-1},
\end{aligned}
$$

or, more briefly, using the conventions of the symbolic (Blissard) calculus,

$$
R_{n}=(t-1) R_{n-1}+(R+R)^{n-1} .
$$

This relation and the boundary condition $R_{0}=1$ completely determine $R_{n}$ and hence the reading numbers $r(x, n)$. Its direct use is clumsy, so we go on to a development.

3. Development of generating function recurrence. Write

$$
\sum_{0}^{\infty} R_{n}(t) u^{n} / n !=\exp u R(t)=\exp u R
$$

in the usual fashion of the symbolic calculus.

Then (3.1) is equivalent to

$$
\frac{d}{d u} \exp u R=(t-1) \exp u R+\exp u(R+R),
$$

or, if $W \equiv W(t, u)=\exp u R$,

$$
d W / d u=(t-1) W+W^{2} .
$$


Noting the boundary condition $W(t, 0)=R_{0}=1$, the solution of this is

$$
W=\exp u R(t)=\frac{1-t}{1-t e^{u(1-t)}},
$$

which is checked by noting that $W(1, u)=(1-u)^{-1}$ since $R_{n}(1)=n$ !

Direct expansion shows that

$$
R_{n}(t)=(1-t)^{n+1} \sum_{0}^{\infty} t^{k} k^{n}
$$

and on using the identity

$$
k^{n}=\sum S(j, n) k(k-1) \cdots(k-j+1)
$$

with $S(j, n)=\Delta^{j} 0^{n} / j !$, a Stirling number, (6) becomes

$$
R_{n}(t)=\sum j ! S(j, n) t^{j}(1-t)^{n-j}
$$

so that

$$
r(x, n)=\sum(-1)^{x-j} C_{n-j, x-j} ! S(j, n) .
$$

This leads immediately to a known result (Dwyer [2], Schrutka [8]) if $j ! S(j, n)$ is replaced by the series obtained by expanding $(E-1)^{j} 0^{n} ;$ namely

$$
\begin{aligned}
r(x, n) & =\sum(-1)^{x-j} C_{n-j, x-i} \sum(-1)^{k} C_{j, k}(k-j)^{n} \\
& =\sum_{0}^{x}(-1)^{j}(x-j)^{n} \sum C_{x-k, j-k} C_{n-x+k, k} \\
& =\sum_{0}^{x}(-1)^{j}(x-j)^{n} C_{n+1, j} .
\end{aligned}
$$

Further, on differentiation, (6) leads to

$$
R_{n+1}(t)=(n+1) t R_{n}+t(1-t) R_{n}^{\prime}(t)
$$

with the prime indicating differentiation. And, by taking coefficients of $t^{x}$, this in turn gives the simplest recurrence formula for the numbers $r(x, n)$, the one which appears naturally in consideration of falls, namely:

$$
r(x, n+1)=x r(x, n)+(n-x+2) r(x-1, n) .
$$

While equation (10) is a natural terminus for the development, it may be noted that $(9)$ (cf. $[4, \S 6])$ implies

$$
R_{n}(1+t)=\sum S(x+1, n+1) x !^{n-x}
$$


which, normalized by a factor $(n !)^{-1}$, is the factorial moment generating function for the density $r(x, n) / n !$.

Finally (5) provides a link between the numbers and a very old expansion; writing $u(1-t)=v$ and $t=e^{s}$, (5) becomes

$$
\exp v \frac{R\left(e^{s}\right)}{1-e^{s}}=\frac{1-e^{s}}{1-e^{s+v}}
$$

so that

$$
R_{n}\left(e^{s}\right)=\frac{1}{\left(1-e^{s}\right)^{n+1}} \frac{d^{n}}{d s^{n}} \frac{1}{1-e^{s}} .
$$

\section{BIBLIOGRAPHY}

1. P.S. Dwyer, The computation of moments with the use of cumulative totals, Ann. Math. Statist. vol. 9 (1938) pp. 288-304.

2. - The cumulative numbers and their polynomials, Ann. Math. Statist. vol. 11 (1940) pp. 66-71.

3. M. F. Gardner and J. L. Barnes, Transients in linear systems, New York, 1942.

4. I. Kaplansky and J. Riordan, The problem of the rooks and its applications, Duke Math. J. vol. 13 (1946) pp. 259-268.

5. A. Sade, Sur les chevauchements des permutations, Marseille, 1949, 8 pp.

6. - Sur les suites hautes des permutations, Marseille, 1949, $12 \mathrm{pp}$.

7. R. Sprague, Über ein Anordnungsproblem, Math. Ann. vol. 121 (1949) pp. 5253.

8. L. v. Schrutka, Eine neue Einteilung der Permutationen, Math. Ann. vol. 118 (1941) pp. 246-250.

Bell TElephone Laboratories 